\title{
New Recursive Representations for the Favard Constants with Application to Multiple Singular Integrals and Summation of Series
}

\author{
Snezhana Georgieva Gocheva-Ilieva and Ivan Hristov Feschiev \\ Faculty of Mathematics and Computer Science, Paisii Hilendarski University of Plovdiv, 24 Tzar Assen Street, 4000 Plovdiv, Bulgaria
}

Correspondence should be addressed to Snezhana Georgieva Gocheva-Ilieva; snegocheva@yahoo.com

Received 11 February 2013; Accepted 22 April 2013

Academic Editor: Josip E. Pecaric

Copyright (C) 2013 S. G. Gocheva-Ilieva and I. H. Feschiev. This is an open access article distributed under the Creative Commons Attribution License, which permits unrestricted use, distribution, and reproduction in any medium, provided the original work is properly cited.

There are obtained integral form and recurrence representations for some Fourier series and connected with them Favard constants. The method is based on preliminary integration of Fourier series which permits to establish general recursion formulas for Favard constants. This gives the opportunity for effective summation of infinite series and calculation of some classes of multiple singular integrals by the Favard constants.

\section{Introduction}

The Fourier series and related with them Achieser-KreinFavard constants, often simply called Favard constants, have significant theoretical and practical roles in many areas $[1$, 2]. These remarkable mathematical constants are introduced firstly in the theory of Fourier series and approximations of functions by trigonometric polynomials [3].

The classical definitions of Favard constants are given by the infinite series $[1,4,5]$

$$
\begin{gathered}
K_{r}=\frac{4}{\pi} \sum_{\nu=0}^{\infty} \frac{(-1)^{\nu(r+1)}}{(2 v+1)^{r+1}}, \quad r=0,1,2, \ldots, \\
\widetilde{K}_{r}=\frac{4}{\pi} \sum_{\nu=0}^{\infty} \frac{(-1)^{\nu r}}{(2 \nu+1)^{r+1}}, \quad r=1,2, \ldots
\end{gathered}
$$

These constants find wide applications in the approximation theory for exact and asymptotic results on the approximation of functions, and especially for the best approximations of trigonometric and other classes of functions in different spaces and related inequalities [1, 5-14]. In particular, many important applications are concerned with the approximation of Euler, cardinal, periodic, and other type of splines [15-17]. It can be noted that Favard constants are connected to approximations that are best in a pointwise sense in comparison, for instance, with the Lebesgue constants which are connected to approximations that are best in a least-squares sense (Fourier series) [5]. The Favard constants play also an important role in estimating optimal quadrature and cubature formulas, calculation of singular integrals, some classes of differential, integrodifferential and integral equations [18-24], and in other areas.

Nevertheless widely used, as a whole, the properties of the Favard constants have not been investigated well enough [14], except for some particular cases.

Different methods for their calculation are given, for instance, in [2, Ch. 5.2]. In general these methods are based on the properties of the well-known constants and special functions as gamma function $\Gamma(z)$, generalized Riemann zeta function $\zeta(z, a)$, the Bernoulli polynomials $B_{n}(x)$ and the Bernoulli numbers $B_{n}$, and the Euler polynomials $E_{n}(x)$ and the Euler numbers $E_{n}$, given by the following expressions [2]:

$$
\begin{gathered}
\Gamma(z)=\int_{0}^{\infty} t^{z-1} e^{-t} d t \quad(\operatorname{Re} z>0), \\
\Psi(z)=\frac{\Gamma^{\prime}(z)}{\Gamma(z)}
\end{gathered}
$$




$$
\begin{gathered}
\zeta(z, a)=\sum_{k=0}^{\infty} \frac{1}{(k+a)^{z}}=\frac{1}{\Gamma(z)} \int_{0}^{\infty} \frac{t^{z-1} e^{-a t}}{1-e^{-t}} d t \quad(\operatorname{Re} z>1), \\
B_{n}(x): \frac{t e^{x t}}{e^{t}-1}=\sum_{k=0}^{\infty} B_{k}(x) \frac{t^{k}}{k !} \quad(|t|<2 \pi), \\
B_{n}=B_{n}(0), \quad B_{n}(x)=\sum_{k=0}^{n}\left(\begin{array}{l}
n \\
k
\end{array}\right) B_{k} x^{n-k}, \\
\left(\frac{1}{\cosh (t)}=\sum_{k=0}^{\infty} E_{k} \frac{t^{k}}{k !}\right), \quad(|t|<\pi) .
\end{gathered}
$$

From now on we will use the following notations:

$$
\begin{gathered}
\widetilde{A}_{n}(x)=\int_{0}^{x} d x \cdots \int_{0}^{x} \ln \tan \frac{\theta}{2} d \theta \quad(0 \leq x \leq \pi), \\
\widetilde{B}_{n}(x)=\int_{0}^{x} d x \cdots \int_{0}^{x} \ln \left(2 \sin \frac{\theta}{2}\right) d \theta \quad(0 \leq x \leq 2 \pi), \\
\widetilde{C}_{n}(x)=\int_{0}^{x} d x \cdots \int_{0}^{x} \ln \left(2 \cos \frac{\theta}{2}\right) d \theta \\
(-\pi \leq x \leq \pi)(n=1,2,3, \ldots) \\
T_{r}=\sum_{n=1}^{\infty} \frac{1}{n^{r}} \quad(r=2,3, \ldots), \\
Q_{r}=\sum_{n=1}^{\infty} \frac{(-1)^{n}}{n^{r}} \quad(x=1,2,3, \ldots),
\end{gathered}
$$

where the three multiple singular integrals (4) contain exactly $n$ integral operations.

The main purpose of this paper is to establish new recursive formulas for the Favard constants (1), including only finite number of terms and recursive formulas for (2). They will be further used to obtain new integral representations for the previously stated objects, in particular for calculation of the multiple singular integrals (4) and summation of series.

\section{Recursive Representations for $K_{r}$ and Some Fourier Series}

We will prove the following.

Theorem 1. For the constants $K_{r}(r=1,2,3, \ldots)$, the following recursive representations hold:

$$
\begin{array}{r}
K_{2 s+1}=\frac{1}{2}\left[(-1)^{s} D_{2 s+1}(\pi)+\sum_{p=1}^{s} K_{2 s+1-2 p} \frac{(-1)^{p-1} \pi^{2 p}}{(2 p) !}\right] \\
\left(K_{0}=1, K_{1}=\frac{\pi}{2}\right), \\
K_{2 s}=(-1)^{s} D_{2 s}\left(\frac{\pi}{2}\right)+\sum_{p=1}^{s} K_{2 s+1-2 p} \frac{(-1)^{p-1}}{(2 p-1) !}\left(\frac{\pi}{2}\right)^{2 p-1} \\
(s=1,2,3, \ldots) .
\end{array}
$$

Proof. We will use the method of induction and preliminary integration of appropriate Fourier series. Let us start by the well-known expansion [2]

$$
\frac{4}{\pi} \sum_{\nu=0}^{\infty} \frac{\sin [(2 \nu+1) x]}{2 \nu+1}=1 \quad(0<x<\pi)
$$

where for $x=\pi / 2$ we will have $K_{0}=1$. By integration of both sides of $(8)$ in $[0, x]$, we get

$$
\begin{aligned}
-\frac{4}{\pi} \sum_{\nu=0}^{\infty} \frac{\cos [(2 \nu+1) x]}{(2 \nu+1)^{2}}+\frac{4}{\pi} \sum_{\nu=0}^{\infty} \frac{1}{(2 \nu+1)^{2}} & =x=D_{1}(x) \\
& (0 \leq x \leq \pi) .
\end{aligned}
$$

For $x=\pi$, (9) gives us $2 K_{1}=\pi=D_{1}(\pi)$, and consequently $K_{1}=D_{1}(\pi) / 2=\pi / 2$. The same results for $K_{0}$ and $K_{1}$ can be achieved starting from the equality [2]

$$
\sum_{n=1}^{\infty} \frac{\sin n x}{n}=\frac{\pi}{2}-\frac{x}{2}=\frac{\pi}{2}-\frac{1}{2} D_{1}(x) \quad(0<x<2 \pi) .
$$

For $x=\pi / 2$ we find again that $K_{0}=1$. After integration of the both sides of (10), we have

$$
\begin{array}{r}
-\sum_{n=1}^{\infty} \frac{\cos n x}{n^{2}}+T_{2}=-\frac{x^{2}}{4}+\frac{\pi x}{2}=-\frac{1}{2} D_{2}(x)+\frac{\pi}{2} D_{1}(x) \\
(0 \leq x \leq 2 \pi) .
\end{array}
$$

For $x=\pi$ we find that $K_{1}=\pi / 2$. Next after integration of the both sides of (11), we obtain

$$
-\sum_{n=1}^{\infty} \frac{\sin n x}{n^{3}}+T_{2} x=-\frac{1}{2} D_{3}(x)+\frac{\pi}{2} D_{2}(x) \quad(0 \leq x \leq 2 \pi),
$$

which for $x=\pi$ gives us

$$
T_{2}=-\frac{1}{2 \pi} D_{3}(\pi)+\frac{1}{2} D_{2}(\pi)=\frac{\pi^{2}}{6}
$$


If we put $x=\pi / 2$ in the same equality (12) and make a little processing, we will arrive at the value $K_{2}=\pi^{2} / 8$.

On the other hand, after integration of both sides of (9), we obtain

$$
-\frac{4}{\pi} \sum_{v=0}^{\infty} \frac{\sin [(2 v+1) x]}{(2 v+1)^{3}}+K_{1} x=D_{2}(x) \quad(0 \leq x \leq \pi) .
$$

Here for $x=\pi / 2$, we get $-K_{2}+(\pi / 2) K_{1}=D_{2}(\pi / 2)$, and consequently

$$
K_{2}=\frac{\pi}{2} K_{1}-D_{2}\left(\frac{\pi}{2}\right)=\frac{\pi^{2}}{8} .
$$

For the constant $K_{3}$, we must now integrate both sides of (12) as

$$
\begin{array}{r}
\sum_{n=1}^{\infty} \frac{\cos n x}{n^{4}}-T_{4}+T_{2} D_{2}(x)=-\frac{1}{2} D_{4}(x)+\frac{\pi}{2} D_{3}(x) \\
(0 \leq x \leq 2 \pi),
\end{array}
$$

and put $x=\pi$ here, or integrate both sides of (14) as

$$
\frac{4}{\pi} \sum_{v=1}^{\infty} \frac{\cos [(2 v+1) x]}{(2 v+1)^{4}}-\frac{4}{\pi} \sum_{v=1}^{\infty} \frac{1}{(2 v+1)^{4}}+K_{1} D_{2}(x)=D_{3}(x)
$$$$
(0 \leq x \leq \pi)
$$

and put again $x=\pi$. Then we will have

$$
\begin{aligned}
K_{3} & =\frac{1}{\pi} D_{4}(\pi)-\frac{3}{2} D_{3}(\pi)+\frac{\pi}{2} D_{2}(\pi) \\
& =\frac{\pi^{2}}{4} K_{1}-\frac{1}{2} D_{3}(\pi)=\frac{\pi^{3}}{24} .
\end{aligned}
$$

Going on the indicated procedure on the base of induction, we easily arrive at the recursive representations ( $7 a$ ) and (7b) and complete the proof.

Remark 2. The scheme of this proof is valid for the most of the other statements in this paper.

In connection with Theorem 1, we would like to note another representation of $K_{r}$ (see, e.g., [25]). It can be written in terms of Lerch transcendent [25], or as it is shown in [2, Section 5.1.4]

$$
\begin{gathered}
K_{2 s-1}=\frac{2}{\pi(2 s) !}\left(2^{2 s}-1\right) \pi^{2 s}\left|B_{2 s}\right|, \\
K_{2 s}=\frac{2}{\pi(2 s) !}\left(\frac{\pi}{2}\right)^{2 s+1}\left|E_{2 s}\right| \quad(s=1,2,3, \ldots),
\end{gathered}
$$

where the Bernoulli and Euler numbers are specified in (3).

Data for values of magnitudes of $K_{r}$ using (7a) and (7b) are shown in Table 1.

The equalities (10)-(13) outline a procedure for summing up the numerical series $T_{2 s},(s=1,2,3, \ldots)$ in (6). It leads to the assertion.
TABLE 1: Exact and approximate values of the Favard constants $K_{r}$, calculated by the recursive formulas (7a) and (7b) using Mathematica software package.

\begin{tabular}{lcc}
\hline$r$ & Exact values of $K_{r}$ & Approximate values of $K_{r}$ \\
\hline 1 & $\pi / 2$ & 1.5707963267948966192313216916 \\
2 & $\pi^{2} / 8$ & 1.2337005501361698273543113749 \\
3 & $\pi^{3} / 24$ & 1.2919281950124925073115131277 \\
4 & $5 \pi^{4} / 384$ & 1.2683475395052400681828168318 \\
5 & $\pi^{5} / 240$ & 1.2750820199386727219280887918 \\
6 & $61 \pi^{6} / 46080$ & 1.2726723265645306132561498711 \\
7 & $17 \pi^{7} / 40320$ & 1.2734371248066831633864461900 \\
8 & $277 \pi^{8} / 2064384$ & 1.2731754806526058136347769671 \\
9 & $31 \pi^{9} / 725760$ & 1.2732612424724875463814366656 \\
10 & $50521 \pi^{10} / 3715891200$ & 1.2732323827293948495082797108 \\
11 & $691 \pi^{11} / 159667200$ & 1.2732419458721540967715077901 \\
12 & $540553 \pi^{12} / 392398110720$ & 1.2732387471572495304117396905 \\
\hline
\end{tabular}

Corollary 3. The following recursive representation holds:

$$
\begin{aligned}
T_{2 s}= & (-1)^{s}\left[\frac{1}{2 \pi} D_{2 s+1}(\pi)-\frac{1}{2} D_{2 s}(\pi)\right] \\
& +\sum_{p=1}^{s-1} \frac{(-1)^{p+1} \pi^{2 p}}{(2 p+1) !} T_{2 s-2 p} \quad(s=1,2,3, \ldots),
\end{aligned}
$$

where for $s=1$ by definition $T_{0}=0$.

It can be noted that the numbers $T_{2 s}$ can be also represented by the well known formula (see, e.g., $[2,5.1 .2]$ )

$$
T_{2 s}=\frac{2^{2 s-1} \pi^{2 s}}{(2 s) !}\left|B_{2 s}\right| \quad(s=1,2,3, \ldots) .
$$

The same procedure applied on the base of the equality

$$
\sum_{n=1}^{\infty}(-1)^{n} \frac{\sin n x}{n}=-\frac{1}{2} D_{1}(x) \quad(-\pi<x<\pi),
$$

leads to the following.

Corollary 4. The following recursive representation holds:

$$
\begin{array}{r}
Q_{2 s}=\frac{(-1)^{s}}{2 \pi} D_{2 s+1}(\pi)+\sum_{p=1}^{s-1} \frac{(-1)^{p+1} \pi^{2 p}}{(2 p+1) !} Q_{2 s-2 p} \\
(s=1,2,3, \ldots),
\end{array}
$$

where for $s=1$ by definition $Q_{0}=0$.

In this connection we will note the explicit formula for $Q_{2 s}$ represented by the Bernoulli numbers (see $[2,5.2 .1]$ )

$$
Q_{2 s}=\frac{\left(1-2^{2 s-1}\right) \pi^{2 s}}{(2 s) !}\left|B_{2 s}\right|, \quad(s=1,2,3, \ldots) .
$$

It is easy to see that the constants $K_{r}$ satisfy the following inequalities (see also [1]):

$$
1=K_{0}<K_{2}<K_{4}<\cdots<\frac{4}{\pi}<\cdots<K_{5}<K_{3}<K_{1}=\frac{\pi}{2}
$$

and $\lim _{r \rightarrow \infty} K_{r}=4 / \pi$. 
The procedure of getting the representations (11), (12), and (16) with the help of (10) gives us an opportunity to lay down the following.

Theorem 5. The following recursive representations hold:

$$
\begin{aligned}
\sum_{n=1}^{\infty} \frac{\sin n x}{n^{2 s-1}}= & (-1)^{s}\left[\frac{1}{2} D_{2 s-1}(x)-\frac{\pi}{2} D_{2 s-2}(x)\right] \\
& +\sum_{p=1}^{s-1}(-1)^{p+1} T_{2 s-2 p} D_{2 p-1}(x), \\
\sum_{n=1}^{\infty} \frac{\cos n x}{n^{2 s}}= & (-1)^{s-1}\left[\frac{1}{2} D_{2 s}(x)-\frac{\pi}{2} D_{2 s-1}(x)\right] \\
& +\sum_{p=0}^{s-1}(-1)^{p} T_{2 s-2 p} D_{2 p}(x)
\end{aligned}
$$

$\left(s=1,2,3, \ldots,(0 \leq x \leq 2 \pi)\right.$, for $s=1$ by definition $D_{0}(x)=1$ and $T_{0}=0,0<x<2 \pi$ ).

At the same time both series in (26) have the well-known representations $[2,5.4 .2]$

$$
\begin{gathered}
\sum_{n=1}^{\infty} \frac{\sin n x}{n^{2 s+1}}=\frac{(-1)^{s-1}}{2(2 s+1) !}(2 \pi)^{2 s+1} B_{2 s+1}\left(\frac{x}{2 \pi}\right), \\
((0 \leq x \leq 2 \pi) ;(s=1,2,3, \ldots), 0<x<2 \pi \text { for } s=0), \\
\sum_{n=1}^{\infty} \frac{\cos n x}{n^{2 s}}=\frac{(-1)^{s-1}}{2(2 s) !}(2 \pi)^{2 s} B_{2 s}\left(\frac{x}{2 \pi}\right) \\
(0 \leq x \leq 2 \pi ; s=1,2,3, \ldots) .
\end{gathered}
$$

The previously stated procedure for obtaining (26) can now be applied on the strength of (22). This leads to the assertion.

Theorem 6. The following recursive representations hold:

$$
\begin{aligned}
\sum_{n=1}^{\infty}(-1)^{n} \frac{\sin n x}{n^{2 s-1}}= & \frac{(-1)^{s}}{2} D_{2 s-1}(x) \\
& +\sum_{p=1}^{s-1}(-1)^{p+1} Q_{2 s-2 p} D_{2 p-1}(x), \\
\sum_{n=1}^{\infty}(-1)^{n} \frac{\cos n x}{n^{2 s}}= & \frac{(-1)^{s-1}}{2} D_{2 s}(x) \\
& +\sum_{p=0}^{s-1}(-1)^{p} Q_{2 s-2 p} D_{2 p}(x),
\end{aligned}
$$

$\left(s=1,2,3, \ldots,(-\pi \leq x \leq \pi)\right.$; for $s=1$ by definition $D_{0}(x)=1$ and $\left.Q_{0}=0,-\pi<x<\pi\right)$.
At the same time, both series in (28) have the well-known representations $[2,5.4 .2]$

$$
\begin{gathered}
\sum_{n=1}^{\infty}(-1)^{n} \frac{\sin n x}{n^{2 s+1}}=\frac{(-1)^{s-1}}{2(2 s+1) !}(2 \pi)^{2 s+1} B_{2 s+1}\left(\frac{x+\pi}{2 \pi}\right) \\
(-\pi<x \leq \pi ; s=0,1,2, \ldots), \\
\sum_{n=1}^{\infty}(-1)^{n} \frac{\cos n x}{n^{2 s}}=\frac{(-1)^{s-1}}{2(2 s) !}(2 \pi)^{2 s} B_{2 s}\left(\frac{x+\pi}{2 \pi}\right) \\
(-\pi \leq x \leq \pi ; s=1,2,3, \ldots) .
\end{gathered}
$$

By analogy with the previous, the procedure for obtaining (9), (14), and (17) with the help of (8) leads us to the following.

Theorem 7. The following recursive representations hold:

$$
\begin{aligned}
& \sum_{v=0}^{\infty} \frac{\sin [(2 v+1) x]}{(2 v+1)^{2 s-1}} \\
& \quad=\frac{\pi(-1)^{s-1}}{4}\left[D_{2 s-2}(x)+\sum_{p=1}^{s-1}(-1)^{p} K_{2 p-1} D_{2 s-2 p-1}(x)\right], \\
& \sum_{v=0}^{\infty} \frac{\cos [(2 v+1) x]}{(2 v+1)^{2 s}} \\
& \quad=\frac{\pi(-1)^{s}}{4}\left[D_{2 s-1}(x)+\sum_{p=1}^{s}(-1)^{p} K_{2 p-1} D_{2 s-2 p}(x)\right],
\end{aligned}
$$

$s=1,2,3, \ldots(0 \leq x \leq \pi)$; for $s=1$ by definition $D_{0}(x)=$ $1(0<x<\pi)$.

At the same time, both series in (30) have the well-known formulas $[2,5.4 .6]$

$$
\begin{aligned}
\sum_{v=0}^{\infty} \frac{\sin [(2 v+1) x]}{(2 v+1)^{2 s+1}}=\frac{(-1)^{s} \pi^{2 s+1}}{4(2 s) !} E_{2 s}\left(\frac{x}{\pi}\right) \\
(0<x<\pi ; s=0,1,2, \ldots), \\
\sum_{v=0}^{\infty} \frac{\cos [(2 v+1) x]}{(2 v+1)^{2 s}}=\frac{(-1)^{s} \pi^{2 s}}{4(2 s-1) !} E_{2 s-1}\left(\frac{x}{\pi}\right) \\
(0 \leq x \leq \pi ; s=1,2, \ldots) .
\end{aligned}
$$

The same procedure applied on the base of the equality

$$
\frac{4}{\pi} \sum_{v=0}^{\infty}(-1)^{v} \frac{\cos [(2 v+1) x]}{2 v+1}=1 \quad\left(-\frac{\pi}{2}<x<\frac{\pi}{2}\right)
$$

gives us the next theorem. 
Theorem 8. The following recursive representations hold:

$$
\begin{aligned}
& \sum_{v=0}^{\infty}(-1)^{v} \frac{\cos [(2 v+1) x]}{(2 v+1)^{2 s-1}} \\
& \quad=\frac{\pi(-1)^{s-1}}{4}\left[D_{2 s-2}(x)+\sum_{p=1}^{s-1}(-1)^{p} K_{2 p} D_{2 s-2 p-2}(x)\right] \\
& \sum_{v=0}^{\infty}(-1)^{v} \frac{\sin [(2 v+1) x]}{(2 v+1)^{2 s}} \\
& \quad=\frac{\pi(-1)^{s-1}}{4}\left[D_{2 s-1}(x)+\sum_{p=1}^{s-1}(-1)^{p} K_{2 p} D_{2 s-2 p-1}(x)\right]
\end{aligned}
$$

$\left(s=1,2,3, \ldots,(-\pi / 2 \leq x \leq \pi / 2)\right.$, for $s=1: K_{0}$ declines, $\left.D_{0}(x)=1,(-\pi / 2<x<\pi / 2)\right)$.

At the same time, both series in (33) have the well-known representations $([2,5.4 .6])$

$$
\begin{aligned}
& \sum_{v=0}^{\infty}(-1)^{v} \frac{\cos [(2 v+1) x]}{(2 v+1)^{2 s+1}}=\frac{(-1)^{s} \pi^{2 s+1}}{4(2 s) !} E_{2 s}\left(\frac{x}{\pi}+\frac{1}{2}\right) \\
&\left(-\frac{\pi}{2}<x<\frac{\pi}{2} ; s=0,1, \ldots\right), \\
& \sum_{v=0}^{\infty}(-1)^{v} \frac{\sin [(2 v+1) x]}{(2 v+1)^{2 s}}=\frac{(-1)^{s-1} \pi^{2 s}}{4(2 s-1) !} E_{2 s-1}\left(\frac{x}{\pi}+\frac{1}{2}\right) \\
&\left(-\frac{\pi}{2} \leq x \leq \frac{\pi}{2} ; s=1,2, \ldots\right) .
\end{aligned}
$$

Meanwhile it is important to note that the number of addends in our recurrence representations (26), (28), (30), and (33) is two times less than the number of the addends in the corresponding cited formulas from [2]. So our method appears to be more economic and effective.

Moreover, one can get many other representations of the constants $K_{r}(r=1,2,3, \ldots)$ and numerical series $Q_{2 s}(s=$ $1,2, \ldots$ ) from Theorems 5-8 putting, in particular, $x=\pi / 2$ or $x=\pi$. For completeness we will note the main results.

From Theorem 5 for $x=\pi / 2$ and $x=\pi$ immediately follows the following.

Corollary 9. For the Favard constants $K_{r}$, the following recursive representations hold:

$$
\begin{aligned}
& K_{2 s-2}=\frac{4}{\pi}\left\{(-1)^{s}\left[\frac{1}{2} D_{2 s-1}\left(\frac{\pi}{2}\right)-\frac{\pi}{2} D_{2 s-2}\left(\frac{\pi}{2}\right)\right]\right. \\
& \left.+\sum_{p=1}^{s-1}(-1)^{p+1} T_{2 s-2 p} D_{2 p-1}\left(\frac{\pi}{2}\right)\right\}, \\
& K_{2 s-1}=\frac{2}{\pi}\left\{(-1)^{s}\left[\frac{1}{2} D_{2 s}(\pi)-\frac{\pi}{2} D_{2 s-1}(\pi)\right]\right. \\
& \left.+\sum_{p=1}^{s-1}(-1)^{p+1} T_{2 s-2 p} D_{2 p}(\pi)\right\}
\end{aligned}
$$

For $x=\pi$, one can get (20) too by replacing previously $s$ by $s+1$.

For $x=\pi / 2$, we obtain the next corollary.

Corollary 10. For numbers $Q_{2 s}$, the following recursive representations hold:

$$
\begin{gathered}
Q_{2 s}=4^{s}\left\{(-1)^{s-1}\left[\frac{1}{2} D_{2 s}\left(\frac{\pi}{2}\right)-\frac{\pi}{2} D_{2 s-1}\left(\frac{\pi}{2}\right)\right]\right. \\
\left.+\sum_{p=0}^{s-1}(-1)^{p} T_{2 s-2 p} D_{2 p}\left(\frac{\pi}{2}\right)\right\}
\end{gathered}
$$

$\left(s=1,2, \ldots\right.$, for $\left.s=1: D_{0}(x)=1\right)$.

Similarly, from Theorem 6 for $x=\pi / 2$ and $x=\pi$, we obtain, respectively, the following.

Corollary 11. For the Favard constants $K_{r}$, the following recursive representations hold:

$$
\begin{aligned}
K_{2 s-2}=\frac{4}{\pi}\left\{\frac{(-1)^{s-1}}{2} D_{2 s-1}\left(\frac{\pi}{2}\right)\right. & \\
& \left.+\sum_{p=1}^{s-1}(-1)^{p} Q_{2 s-2 p} D_{2 p-1}\left(\frac{\pi}{2}\right)\right\}, \\
K_{2 s-1}=\frac{2}{\pi}\left\{\frac{(-1)^{s-1}}{2} D_{2 s}(\pi)\right. & \\
& \left.+\sum_{p=1}^{s-1}(-1)^{p} Q_{2 s-2 p} D_{2 p}(\pi)\right\},
\end{aligned}
$$

where $s=1,2, \ldots$, and for $s=1: D_{0}(x)=1, Q_{0}=0$.

For $x=\pi$, one can get (23) too by replacing previously $s$ by $s+1$.

For $x=\pi / 2$ from Theorem 6 , (the second formula in (28)), we will have also the next analogous corollary.

Corollary 12. For numbers $Q_{2 s}$, the following recursive representations hold:

$$
\begin{aligned}
Q_{2 s}=\frac{4^{s}}{4^{s}-1}\{ & \frac{(-1)^{s}}{2} D_{2 s}\left(\frac{\pi}{2}\right) \\
& \left.+\sum_{p=1}^{s-1}(-1)^{p+1} Q_{2 s-2 p} D_{2 p}\left(\frac{\pi}{2}\right)\right\},
\end{aligned}
$$

$\left(s=1,2, \ldots\right.$, for $\left.s=1: D_{0}(x)=1, Q_{0}=0\right)$.

By the same manner from Theorem 7 for $x=\pi$ and $x=\pi / 2$, we obtain, respectively, the formulas for $K_{r}(r=$ $1,2,3, \ldots)$ different from these in Theorem 1 . 
Corollary 13. For the Favard constants $K_{2 s-3}$ and $K_{2 s-1}$, the following recursive representations hold:

$$
K_{2 s-3}=\frac{(-1)^{s}}{\pi}\left\{D_{2 s-2}(\pi)+\sum_{p=1}^{s-2}(-1)^{p} K_{2 p-1} D_{2 s-2 p-1}(\pi)\right\},
$$

$\left(s=2,3, \ldots\right.$, for $s=2, K_{1} D_{1}(\pi)$ must be canceled $)$ and

$K_{2 s-1}=(-1)^{s-1}\left\{D_{2 s-1}\left(\frac{\pi}{2}\right)+\sum_{p=1}^{s-1}(-1)^{p} K_{2 p-1} D_{2 s-2 p}\left(\frac{\pi}{2}\right)\right\}$,

$\left(s=1,2,3, \ldots\right.$, for $s=1, K_{1} D_{0}(\pi / 2)$ must be canceled $)$.

The remaining cases for $x=\pi / 2$ and $x=\pi$ immediately lead to Theorem 1 after replacing $s$ by $s+1$.

From Theorem 8 for $x=\pi / 2$, one can get, respectively, other representations for $K_{r}(r=1,2,3, \ldots)$, different from these in Theorem 1.

Corollary 14. For the Favard constants $K_{r}$, the following recursive representations hold:

$$
\begin{gathered}
K_{2 s-2}=(-1)^{s}\left\{D_{2 s-2}\left(\frac{\pi}{2}\right)+\sum_{p=1}^{s-2}(-1)^{p} K_{2 p} D_{2 s-2 p-2}\left(\frac{\pi}{2}\right)\right\}, \\
K_{2 s-1}=(-1)^{s-1}\left\{D_{2 s-1}\left(\frac{\pi}{2}\right)+\sum_{p=1}^{s-1}(-1)^{p} K_{2 p} D_{2 s-2 p-1}\left(\frac{\pi}{2}\right)\right\},
\end{gathered}
$$

where $s=1,2,3, \ldots$, for $s=1: D_{0}(\pi / 2)=-1$, and for $s=2$ : $K_{2} D_{0}(\pi / 2)$ must be canceled.

Corollary 15. From the difference $T_{2 s}-Q_{2 s}=(\pi / 2) K_{2 s-1}(s=$ $1,2, \ldots)$ and after replacing s by $s+1$ in the obtained expression, one gets the following formula:

$$
\begin{array}{r}
K_{2 s+1}=\frac{(-1)^{s}}{\pi} D_{2 s+2}(\pi)+\sum_{p=1}^{s} \frac{(-1)^{p+1} \pi^{2 p}}{(2 p+1) !} K_{2 s-2 p+1} \\
(s=0,1, \ldots) .
\end{array}
$$

This is somewhat better than the corresponding formula in Theorem 1, because $(2 s+1) !>2(2 s)$ ! for $s=1,2, \ldots$.

\section{Recursive Representations for $\widetilde{K}_{r}$ and Some Fourier Series}

Here we will get down to the integral representation and recursive formulas for the constants $\widetilde{K}_{r}$, defined in (2). As one can see they are closely linked with the approximation of the conjugate classes of functions obtained on the base of the Hilbert transform $[1,26]$. First of all let us note their representations easily obtained by means of special functions in (3) as it is shown in $[2,5.1 .4]$ for the Catalan constant as follows:

$$
\widetilde{K}_{r}=\left\{\begin{array}{cc}
\frac{4}{\pi}\left(1-2^{-(2 s+1)}\right) \zeta(2 s+1), & r=2 s, s=1,2,3, \ldots, \\
\frac{2^{2-4 s}}{\pi}\left(\zeta\left(2 s, \frac{1}{4}\right)-\zeta\left(2 s, \frac{3}{4}\right)\right) & \\
=\frac{2}{\pi \Gamma(2 s)} \int_{0}^{\infty} \frac{t^{2 s-1}}{\operatorname{ch} t} d t, & r=2 s-1, s=1,2,3, \ldots
\end{array}\right.
$$

In the beginning we will prove the following assertion.

Theorem 16. For the constants $\widetilde{K}_{r}(r=1,2, \ldots)$, the following recursive representations hold:

$$
\begin{gathered}
\widetilde{K}_{2 s-1}=\frac{2(-1)^{s}}{\pi} \widetilde{A}_{2 s-1}\left(\frac{\pi}{2}\right)+\sum_{p=1}^{s-1} \widetilde{K}_{2 s-2 p} \frac{(-1)^{p-1}}{(2 p-1) !}\left(\frac{\pi}{2}\right)^{2 p-1}, \\
\widetilde{K}_{2 s}=\frac{2(-1)^{s}}{\pi} \widetilde{A}_{2 s}\left(\frac{\pi}{2}\right)+\sum_{p=1}^{s-1} \widetilde{K}_{2 s-2 p} \frac{(-1)^{p-1}}{(2 p) !}\left(\frac{\pi}{2}\right)^{2 p} \\
\left(s=1,2,3, \ldots ; \widetilde{K}_{0} \stackrel{\text { def }}{=} 0\right) .
\end{gathered}
$$

Proof. The proof of this theorem is based on induction again. However, for completeness we must give somewhat more detailed considerations at first steps, which underline further discussions.

Let us start by the well-known Fourier expansions (see, e.g., $[2,5.4])$

$$
\begin{gathered}
\sum_{v=0}^{\infty} \frac{\sin [(2 v+1) x]}{(2 v+1)^{2}}=-\frac{1}{2} \widetilde{A}_{1}(x) \quad(0 \leq x \leq \pi), \\
\sum_{n=1}^{\infty} \frac{\sin n x}{n^{2}}=-\widetilde{B}_{1}(x) \quad(0 \leq x \leq 2 \pi) .
\end{gathered}
$$

For $x=\pi / 2$, the first equality gives us immediately $(\pi /$ 4) $\widetilde{K}_{1}=-(1 / 2) \widetilde{A}_{1}(\pi / 2)$. At the same time, the second equality in (45) leads to $(\pi / 4) \widetilde{K}_{1}=-\widetilde{B}_{1}(\pi / 2)$. So we obtain the integral representation of $\widetilde{K}_{1}$ in the form

$$
\widetilde{K}_{1}=-\frac{4}{\pi} \widetilde{B}_{1}\left(\frac{\pi}{2}\right)=-\frac{2}{\pi} \widetilde{A}_{1}\left(\frac{\pi}{2}\right)=1.166243616123275 \cdots .
$$

We have another integral representation of the same constant $\widetilde{K}_{1}$ in our paper [8].

Further by integration of both sides of the first equality in (45), we get

$$
\sum_{v=0}^{\infty} \frac{\cos [(2 v+1) x]}{(2 v+1)^{3}}=\frac{1}{2} \widetilde{A}_{2}(x)+\frac{\pi}{4} \widetilde{K}_{2} \quad(0 \leq x \leq \pi),
$$


from where for $x=\pi / 2$ and $x=\pi$ we have simultaneously $0=(1 / 2) \widetilde{A}_{2}(\pi / 2)+(\pi / 4) \widetilde{K}_{2}$ and $-(\pi / 4) \widetilde{K}_{2}=(1 / 2) \widetilde{A}_{2}(\pi)+$ $(\pi / 4) \widetilde{K}_{2}$, and consequently

$$
\widetilde{K}_{2}=-\frac{2}{\pi} \widetilde{A}_{2}\left(\frac{\pi}{2}\right)=-\frac{1}{\pi} \widetilde{A}_{2}(\pi) .
$$

Then, after the integration of both sides of the second equality in (45), we obtain

$$
\sum_{n=1}^{\infty} \frac{\cos n x}{n^{3}}=\widetilde{B}_{2}(x)+T_{3} \quad(0 \leq x \leq 2 \pi),
$$

from where for $x=\pi$ we have $(\pi / 2) \widetilde{K}_{2}=-\widetilde{B}_{2}(\pi)$. If we correspond this with (48), we find

$$
\begin{aligned}
\widetilde{K}_{2} & =-\frac{2}{\pi} \widetilde{B}_{2}(\pi)=-\frac{2}{\pi} \widetilde{A}_{2}\left(\frac{\pi}{2}\right) \\
& =-\frac{1}{\pi} \widetilde{A}_{2}(\pi)=1.339193086109090 \cdots
\end{aligned}
$$

In order to obtain the integral representation of $\widetilde{K}_{3}$, we must, at first, integrate the both sides of (47) as

$$
\sum_{v=0}^{\infty} \frac{\sin [(2 v+1) x]}{(2 v+1)^{4}}=\frac{1}{2} \widetilde{A}_{3}(x)+\frac{\pi}{4} \widetilde{K}_{2} D_{1}(x) \quad(0 \leq x \leq \pi) .
$$

Next it remains to put $x=\pi / 2$ as

$$
\widetilde{K}_{3}=\frac{2}{\pi} \widetilde{A}_{3}\left(\frac{\pi}{2}\right)+\frac{\pi}{2} \widetilde{K}_{2}=\frac{2}{\pi} \widetilde{A}_{3}\left(\frac{\pi}{2}\right)-\widetilde{A}_{2}\left(\frac{\pi}{2}\right) .
$$

As another integral representation of $\widetilde{K}_{3}$, we can get after integration of both sides of (49)

$$
\sum_{n=1}^{\infty} \frac{\sin n x}{n^{4}}=\widetilde{B}_{3}(x)+T_{3} D_{1}(x) \quad(0 \leq x \leq 2 \pi) .
$$

On one hand, for $x=\pi$, (53) gives

$$
T_{3}=-\frac{1}{\pi} \widetilde{B}_{3}(\pi)=1.20205690 \cdots
$$

On the other hand, the same equality (53) for $x=\pi / 2$ gives $\sum_{v=0}^{\infty} \sin [(2 v+1) \pi / 2] /(2 v+1)^{4}=\widetilde{B}_{3}(\pi / 2)-(1 / 2) \widetilde{B}_{3}(\pi)$. Then

$$
\begin{aligned}
\widetilde{K}_{3} & =\frac{4}{\pi} \widetilde{B}_{3}\left(\frac{\pi}{2}\right)-\frac{2}{\pi} \widetilde{B}_{3}(\pi)=\frac{2}{\pi} \widetilde{A}_{3}\left(\frac{\pi}{2}\right)-\widetilde{A}_{2}\left(\frac{\pi}{2}\right) \\
& =1.259163310827165 \cdots .
\end{aligned}
$$

For the constant $\widetilde{K}_{4}$ there are also different ways to receive its integral representations. One of them is based on the integration of both sides of (53) as

$$
-\sum_{n=1}^{\infty} \frac{\cos n x}{n^{5}}+T_{5}=\widetilde{B}_{4}(x)+T_{3} D_{2}(x) \quad(0 \leq x \leq 2 \pi) .
$$

For $x=\pi,(56)$ gives $(\pi / 2) \widetilde{K}_{4}=\widetilde{B}_{4}(\pi)+\left(\pi^{2} / 2\right) T_{3}$. Admitting (54) we will have

$$
\widetilde{K}_{4}=\frac{2}{\pi} \widetilde{B}_{4}(\pi)-\widetilde{B}_{3}(\pi)=1.278999378416936 \cdots .
$$

Another integral representation of $\widetilde{K}_{4}$ can be obtained by integration of both sides of (51) as

$$
-\sum_{v=0}^{\infty} \frac{\cos [(2 v+1) x]}{(2 v+1)^{5}}+\frac{\pi}{4} \widetilde{K}_{4}=\frac{1}{2} \widetilde{A}_{4}(x)+\frac{\pi}{4} \widetilde{K}_{2} D_{2}(x)
$$

$$
(0 \leq x \leq \pi) .
$$

For $x=\pi / 2$ and $x=\pi$, we get, respectively,

$$
\begin{aligned}
\widetilde{K}_{4} & =\frac{2}{\pi} \widetilde{A}_{4}\left(\frac{\pi}{2}\right)-\frac{\pi}{4} \widetilde{A}_{2}\left(\frac{\pi}{2}\right)=\frac{2}{\pi} \widetilde{A}_{4}\left(\frac{\pi}{2}\right)+\frac{\pi^{2}}{8} \widetilde{K}_{2} \\
& =\frac{1}{\pi} \widetilde{A}_{4}(\pi)+\frac{\pi^{2}}{4} \widetilde{K}_{2} .
\end{aligned}
$$

Further after integration of both sides of (56), we get

$-\sum_{v=0}^{\infty} \frac{\sin n x}{n^{6}}+T_{5} D_{1}(x)=\widetilde{B}_{5}(x)+T_{3} D_{3}(x) \quad(0 \leq x \leq 2 \pi)$.

On one hand, if we put here $x=\pi$ and admit (54), we will have

$$
\begin{aligned}
T_{5} & =\frac{1}{\pi} \widetilde{B}_{5}(\pi)+\frac{\pi^{2}}{3 !} T_{3}=\frac{1}{\pi} \widetilde{B}_{5}(\pi)-\frac{\pi}{6} \widetilde{B}_{3}(\pi) \\
& =1.03692776 \cdots .
\end{aligned}
$$

On the other hand, the same formula (60) for $x=\pi / 2$ gives us $-(\pi / 4) \widetilde{K}_{5}+(\pi / 2) T_{5}=\widetilde{B}_{5}(\pi / 2)+\left(\pi^{3} / 2^{3} 3 !\right) T_{3}$. Then admitting (54) and (61), we obtain

$$
\begin{aligned}
\widetilde{K}_{5} & =-\frac{\pi}{4} \widetilde{B}_{5}\left(\frac{\pi}{2}\right)+\frac{2}{\pi} \widetilde{B}_{5}(\pi)-\frac{\pi}{4} \widetilde{B}_{3}(\pi) \\
& =1.271565517671139 \cdots .
\end{aligned}
$$

Next, in order to receive another integral representation of $\widetilde{K}_{5}$, we must integrate both sides of (58). So we will have

$-\sum_{v=0}^{\infty} \frac{\sin [(2 v+1) x]}{(2 v+1)^{6}}+\frac{\pi}{4} \widetilde{K}_{4} D_{1}(x)=\frac{1}{2} \widetilde{A}_{2}(x)+\frac{\pi}{4} \widetilde{K}_{2} D_{3}(x)$ $(0 \leq x \leq \pi)$.

Putting $x=\pi / 2$, here, we get

$$
\begin{aligned}
\widetilde{K}_{5} & =-\frac{2}{\pi} \widetilde{A}_{5}\left(\frac{\pi}{2}\right)+\frac{\pi}{2} \widetilde{K}_{4}-\frac{\pi^{3}}{2^{3} 3 !} \widetilde{K}_{2} \\
& =-\frac{2}{\pi} \widetilde{A}_{5}\left(\frac{\pi}{2}\right)+\widetilde{A}_{4}\left(\frac{\pi}{2}\right)-\frac{\pi^{2}}{2^{2} \cdot 3} \widetilde{A}_{2}\left(\frac{\pi}{2}\right) .
\end{aligned}
$$

Now after this preparatory work, we can go on the indicated procedure which leads us to the general recursive representations (43) and so complete the proof. 
The previously stated procedure gives us the opportunity to obtain recursive formulas for the multiple integrals $\widetilde{A}_{r}, \widetilde{B}_{r}$, and $\widetilde{C}_{r}$ in (4) for a given $x$.

First of all we will note that on the base of induction and with the help of the first formula in (45), (47), (51), (58), and (63), we can lay down the following.

Theorem 17. For the multiple singular integrals $\widetilde{A}_{r}$, the following recursive representations hold:

$$
\begin{aligned}
\widetilde{A}_{2 s-1}(x)= & 2(-1)^{s} \sum_{v=0}^{\infty} \frac{\sin [(2 v+1) x]}{(2 v+1)^{2 s}} \\
& +\frac{\pi}{2} \sum_{p=1}^{s-1}(-1)^{p} \widetilde{K}_{2 p} D_{2 s-2 p-1}(x), \\
\widetilde{A}_{2 s}(x)= & -2(-1)^{s} \sum_{v=0}^{\infty} \frac{\cos [(2 v+1) x]}{(2 v+1)^{2 s+1}} \\
& +\frac{\pi}{2} \sum_{p=1}^{s}(-1)^{p} \widetilde{K}_{2 p} D_{2 s-2 p}(x),
\end{aligned}
$$

$s=1,2,3, \ldots(0 \leq x \leq \pi), D_{0}=1$, for $s=0: \widetilde{A}_{0}(x)=$ $\ln (\tan (x / 2))(0<x<\pi)$, and $\widetilde{K}_{0}=0$, where

$$
\begin{aligned}
\sum_{v=0}^{\infty} \frac{\sin [(2 v+1) x]}{(2 v+1)^{2 s}} & =\frac{1}{2}\left\{\sum_{n=1}^{\infty} \frac{\sin n x}{n^{2 s}}-\sum_{n=1}^{\infty}(-1)^{n} \frac{\sin n x}{n^{2 s}}\right\} \\
& =\frac{1}{\Gamma(2 s)} \int_{0}^{\infty} \frac{t^{2 s-1} e^{t}\left(e^{2 t}+1\right) \sin x}{\left(e^{2 t}+1\right)^{2}-4 e^{2 t} \cos ^{2} x} d t \\
\sum_{v=0}^{\infty} \frac{\cos [(2 v+1) x]}{(2 v+1)^{2 s+1}} & =\frac{1}{2}\left\{\sum_{n=1}^{\infty} \frac{\cos n x}{n^{2 s+1}}-\sum_{n=1}^{\infty}(-1)^{n} \frac{\cos n x}{n^{2 s+1}}\right\} \\
& =\frac{1}{\Gamma(2 s+1)} \int_{0}^{\infty} \frac{t^{2 s} e^{t}\left(e^{2 t}-1\right) \cos x}{\left(e^{2 t}+1\right)^{2}-4 e^{2 t} \cos ^{2} x} d t
\end{aligned}
$$

By analogy with the previous and by means of the second formula in (45), (49), (53), (56), and (60), one can get the following.

Theorem 18. For the multiple singular integrals $\widetilde{B}_{r}$, the following recursive representations hold:

$$
\begin{aligned}
& \widetilde{B}_{2 s-1}(x) \\
& =(-1)^{s}\left\{\sum_{n=1}^{\infty} \frac{\sin n x}{n^{2 s}}+\sum_{p=1}^{s-1}(-1)^{p} T_{2 s-2 p+1} D_{2 p-1}(x)\right\}, \\
& \widetilde{B}_{2 s-2}(x) \\
& =(-1)^{s}\left\{\sum_{n=1}^{\infty} \frac{\cos n x}{n^{2 s-1}}+\sum_{p=1}^{s-1}(-1)^{p} T_{2 s-2 p+1} D_{2 p-2}(x)\right\},
\end{aligned}
$$

$s=1,2,3, \ldots(0 \leq x \leq 2 \pi), D_{0}=1$, for $s=1: T_{1} D_{k}(k=0,1)$ declines, by definition $\widetilde{B}_{0}(x)=\ln (2 \sin (x / 2))(0<x<2 \pi)$, where (see [2])

$$
\begin{gathered}
\sum_{n=1}^{\infty} \frac{\sin n x}{n^{2 s}}=\frac{1}{\Gamma(2 s)} \int_{0}^{\infty} \frac{t^{2 s-1} e^{t} \sin x}{1-2 e^{t} \cos x+e^{2 t}} d t \\
(x \neq 0, s=1,2,3, \ldots), \\
\sum_{n=1}^{\infty} \frac{\cos n x}{n^{2 s-1}}=\frac{1}{\Gamma(2 s-1)} \int_{0}^{\infty} \frac{t^{2 s-2}\left(e^{t} \cos x-1\right)}{1-2 e^{t} \cos x+e^{2 t}} d t \\
\quad(x \neq 0, s=1,2,3, \ldots) .
\end{gathered}
$$

As similar representations of $\widetilde{C}_{r}(x)$, one can get on the base of the expansion (see $[2,5.4 .2]$ )

$$
\begin{gathered}
\sum_{n=1}^{\infty}(-1)^{n} \frac{\cos n x}{n}=-\ln \left(2 \cos \left(\frac{x}{2}\right)\right) \quad(-\pi<x<\pi), \\
Q_{1}=-\ln 2 .
\end{gathered}
$$

Theorem 19. For the multiple singular integrals $\widetilde{C}_{r}$, the following recursive representations hold:

$$
\begin{array}{r}
\widetilde{C}_{2 s-1}(x) \\
=(-1)^{s}\left\{\sum_{n=1}^{\infty}(-1)^{n} \frac{\sin n x}{n^{2 s}}+\sum_{p=1}^{s-1}(-1)^{p} Q_{2 s-2 p+1} D_{2 p-1}(x)\right\}, \\
\widetilde{C}_{2 s-2}(x) \\
=(-1)^{s}\left\{\sum_{n=1}^{\infty}(-1)^{n} \frac{\cos n x}{n^{2 s-1}}+\sum_{p=1}^{s-1}(-1)^{p} Q_{2 s-2 p+1} D_{2 p-2}(x)\right\},
\end{array}
$$

$s=1,2,3, \ldots(-\pi \leq x \leq \pi)$, for $s=1: \widetilde{C}_{0}(x)=\ln (2 \cos (x)$

2)) $(-\pi<x<\pi), Q_{1} D_{k}(k=0,1)$ declines, where (see [2])

$$
\begin{array}{r}
\sum_{n=1}^{\infty}(-1)^{n} \frac{\sin n x}{n^{2 s}}=\frac{-1}{\Gamma(2 s)} \int_{0}^{\infty} \frac{t^{2 s-1} e^{t} \sin x}{1+2 e^{t} \cos x+e^{2 t}} d t \\
(s=1,2,3, \ldots), \\
\sum_{n=1}^{\infty}(-1)^{n} \frac{\cos n x}{n^{2 s-1}}=\frac{-1}{\Gamma(2 s-1)} \int_{0}^{\infty} \frac{t^{2 s-2}\left(e^{t} \cos x+1\right)}{1+2 e^{t} \cos x+e^{2 t}} d t \\
(s=1,2, \ldots) .
\end{array}
$$

By analogy with the previous, if we start from the expansion $[2,5.4 .6]$

$$
\begin{array}{r}
\sum_{v=0}^{\infty}(-1)^{v} \frac{\sin [(2 v+1) x]}{2 v+1}=\frac{-1}{2} \ln \left(\tan \left(\frac{\pi}{4}-\frac{x}{2}\right)\right) \\
\left(-\frac{\pi}{2}<x<\frac{\pi}{2}\right),
\end{array}
$$

we can obtain the next theorem. 
Theorem 20. The following recursive formulas hold:

$$
\begin{aligned}
\int_{0}^{\pi / 2-x} \widetilde{A}_{2 s-1}(u) d u= & -2(-1)^{s} \sum_{v=0}^{\infty}(-1)^{v} \frac{\sin [(2 v+1) x]}{(2 v+1)^{2 s+1}} \\
& +\frac{\pi}{2} \sum_{p=1}^{s}(-1)^{p} \widetilde{K}_{2 p} D_{2 s-2 p}\left(\frac{\pi}{2}-x\right) \\
\int_{0}^{\pi / 2-x} \widetilde{A}_{2 s-2}(u) d u= & 2(-1)^{s} \sum_{v=0}^{\infty}(-1)^{v} \frac{\cos [(2 v+1) x]}{(2 v+1)^{2 s}} \\
& -\frac{\pi}{2} \sum_{p=1}^{s-1}(-1)^{p} \widetilde{K}_{2 p} D_{2 s-2 p-1}\left(\frac{\pi}{2}-x\right)
\end{aligned}
$$

$s=1,2,3, \ldots(-\pi / 2 \leq x \leq \pi / 2), D_{0}(x)=1$, for $s=1$ : $\widetilde{A_{0}}(u)=\ln \tan (u / 2)(-\pi / 2<u<\pi / 2)$, where

$$
\begin{aligned}
& \sum_{v=0}^{\infty}(-1)^{v} \frac{\sin [(2 v+1) x]}{(2 v+1)^{2 s+1}} \\
& =\frac{1}{\Gamma(2 s+1)} \int_{0}^{\infty} \frac{t^{2 s} e^{t}\left(e^{2 t}-1\right) \sin x}{\left(e^{2 t}+1\right)^{2}-4 e^{2 t} \sin ^{2} x} d t \\
& (s=1,2, \ldots),
\end{aligned}
$$

$$
\begin{aligned}
& \sum_{v=0}^{\infty}(-1)^{v} \frac{\cos [(2 v+1) x]}{(2 v+1)^{2 s}} \\
&=\frac{1}{\Gamma(2 s)} \int_{0}^{\infty} \frac{t^{2 s-1} e^{t}\left(e^{2 t}+1\right) \cos x}{\left(e^{2 t}+1\right)^{2}-4 e^{2 t} \sin ^{2} x} d t \\
&(s=1,2, \ldots) .
\end{aligned}
$$

The proof of this theorem requires to take integration from $\pi / 2$ to $x$ and then to make the substitution $t=\pi / 2-x$.

From Table 2 one can see that $\widetilde{A}_{r}(x)=\widetilde{B}_{r}(x)-\widetilde{C}_{r}(x)(0 \leq$ $x \leq \pi), r=1,2, \ldots$. The constants $\widetilde{K}_{r}$ satisfy the following inequalities [1]:

$$
1<\widetilde{K}_{1}<\widetilde{K}_{3}<\widetilde{K}_{5}<\cdots<\frac{4}{\pi}<\cdots<\widetilde{K}_{6}<\widetilde{K}_{4}<\widetilde{K}_{2}<\frac{\pi}{2} .
$$

Remark 21. The equalities (49), (53), (56), and (60) outline a procedure for summing up the numerical series $T_{2 s+1}(s=$ $1,2, \ldots)$ in (2). It leads us to the following.

Corollary 22. The following recursive representation holds:

$$
T_{2 s+1}=\frac{(-1)^{s}}{\pi} \widetilde{B}_{2 s+1}(\pi)+\sum_{p=1}^{s-1}(-1)^{p+1} \frac{\pi^{2 p}}{(2 p+1) !} T_{2 s-2 p+1}
$$

$s=1,2, \ldots$ for $s=1$ the term $\left(\pi^{2} / 6\right) T_{1}$ declines.
For comparison we will note also the well-known formula $[2,5.1 .2]$

$$
\begin{aligned}
T_{2 s+1} & =\zeta(2 s+1)=\frac{1}{\Gamma(2 s+1)} \int_{0}^{\infty} \frac{t^{2 s}}{e^{t}-1} d t \\
& =\frac{-1}{(2 s) !} \Psi^{(2 s)}(1) \quad(s=1,2, \ldots) .
\end{aligned}
$$

The same procedure based on induction and preliminary multiple integration of both sides of (69) leads us to the next.

Corollary 23. The following recursive representation holds:

$$
Q_{2 s+1}=\frac{(-1)^{s}}{\pi} \widetilde{C}_{2 s+1}(\pi)+\sum_{p=1}^{s-1}(-1)^{p+1} \frac{\pi^{2 p}}{(2 p+1) !} Q_{2 s-2 p+1},
$$

$s=1,2, \ldots$, for $s=1$ the term $\left(\pi^{2} / 6\right) Q_{1}$ declines.

As in previous, let us give the alternate formula [2, 5.1.3]

$$
\begin{array}{r}
Q_{2 s+1}=\left(2^{-2 s}-1\right) \zeta(2 s+1)=\frac{-1}{\Gamma(2 s+1)} \int_{0}^{\infty} \frac{t^{2 s}}{e^{t}+1} d t \\
(s=1,2, \ldots) .
\end{array}
$$

At the end we would like to note also that one can get many other representations (through multiple integrals) of the constants $\widetilde{K}_{r}(r=1,2, \ldots)$ from Theorems 17-20 setting, in particular, $x=\pi / 2$ or $x=\pi$, as it is made for the constants $K_{r}$.

From the difference $T_{2 s+1}-Q_{2 s+1}=(\pi / 2) \widetilde{K}_{2 s}(s=1,2$, $\ldots)$, one can get the following formula:

$$
\begin{array}{r}
\widetilde{K}_{2 s}=\frac{2(-1)^{s}}{\pi^{2}} \widetilde{A}_{2 s+1}(\pi)+\sum_{p=1}^{s-1}(-1)^{p+1} \frac{\pi^{2 p}}{(2 p+1) !} \widetilde{K}_{2 s-2 p} \\
(s=1,2, \ldots),
\end{array}
$$

which is somewhat inferior to the similar recursion formula in Theorem 16 because $(2 s-1) ! \leq 2^{2 s-2}(2 s-2)$ ! for $s=1,2, \ldots$.

As an exception we will give the inverse formula of (80) as

$$
\begin{array}{r}
\widetilde{A}_{2 s+1}(\pi)=(-1)^{s} \sum_{p=1}^{s}(-1)^{p+1} \frac{\pi^{2 p}}{2(2 p-1) !} \widetilde{K}_{2 s-2 p+2} \\
(s=1,2, \ldots) .
\end{array}
$$

If we, by definition, lay $\widetilde{K}_{0}=0$, then the equality (80) is valid for $s=0$ too.

\section{Some Notes on Numerical and Computer Implementations of the Derived Formulas}

We will consider some aspects of numerical and symbolic calculations of the Favard constants $K_{r}$, singular integrals (4), and summation of series. 
TABLE 2: Calculated values of the magnitudes $\widetilde{A}_{r}(x), \widetilde{B}_{r}(x)$, and $\widetilde{C}_{r}(x)$ for $x=\pi / 2, \pi$, by using formulas (65), (67), and (70), respectively.

\begin{tabular}{|c|c|c|c|}
\hline$r$ & $\widetilde{A}_{r}(\pi / 2)$ & $\widetilde{B}_{r}(\pi / 2)$ & $\widetilde{C}_{r}(\pi / 2)$ \\
\hline 0 & & 0.346573590279972654709 & 0.34657359027997265471 \\
\hline 1 & -1.831931188354438030 & -0.91596559417721901505 & 0.91596559417721901506 \\
\hline 2 & -2.103599580529289999 & -1.31474973783080624966 & 0.78884984269848374979 \\
\hline 3 & -1.326437390660483389 & -0.89924201634043412749 & 0.42719537432004926159 \\
\hline 4 & -0.586164434174921923 & -0.41567176475313624651 & 0.17049266942178567636 \\
\hline 5 & -0.200415775434410589 & -0.14636851588819525831 & 0.05404725954621533063 \\
\hline 6 & -0.055999130148030908 & -0.04177075772178286763 & 0.01422837242624804042 \\
\hline 7 & -0.013242890305882861 & -0.01003832077074076074 & 0.00320456953514210000 \\
\hline 8 & -0.002716221784223860 & -0.00208540018388810697 & 0.00063082160033575329 \\
\hline 9 & -0.000492033289602442 & -0.00038173093475665008 & 0.00011030235484579206 \\
\hline 10 & -0.000079824623761109 & -0.00006247448634651347 & 0.00001735013741459518 \\
\hline 11 & -0.000011727851308441 & -0.00000924763886476940 & 0.00000248021244367167 \\
\hline 12 & -0.000001574609462951 & -0.00000124968150024699 & 0.00000032492796270360 \\
\hline$r$ & $\widetilde{A}_{r}(\pi)$ & $\widetilde{B}_{r}(\pi)$ & $\widetilde{C}_{r}(\pi)$ \\
\hline 0 & & 0.693147180559945309417 & \\
\hline 1 & 0 & 0 & 0 \\
\hline 2 & -4.2071991610585799989 & -2.10359958052928999945 & 2.10359958052928999945 \\
\hline 3 & -6.6086529882853881226 & -3.77637313616307892720 & 2.83227985212230919540 \\
\hline 4 & -6.3627527878802461560 & -3.92286552530160912232 & 2.43988726257863703368 \\
\hline 5 & -4.5591894893017946564 & -2.95428020294335768590 & 1.60490928635843697049 \\
\hline 6 & -2.6255391709472096141 & -1.76271265891422894113 & 0.86282651203298067295 \\
\hline 7 & -1.2684915655900274347 & -0.87472015815662857450 & 0.39377140743339886016 \\
\hline 8 & -0.5287656648068309189 & -0.37237211738940254175 & 0.15639354741742837714 \\
\hline 9 & -0.1940031559506448617 & -0.13897143291435930434 & 0.05503172303628555739 \\
\hline 10 & -0.0635985732918145806 & -0.04620733330628432785 & 0.01739123998553025277 \\
\hline 11 & -0.0188489390019113804 & -0.01385968931040814707 & 0.00498924969150323336 \\
\hline 12 & -0.0050985327885974909 & -0.00378779048977775268 & 0.00131074229881973820 \\
\hline
\end{tabular}

Let us take only the first $m-1, m \leq s+1$ terms in the final sums in the right-hand side of the formulas (7a) and (7b) and denote the remaining truncation sums by

$$
\begin{gathered}
S_{1, m}=\sum_{j=m}^{s} \frac{(-1)^{j-1} \pi^{j p}}{(2 j) !} K_{2 s-2 j+1}, \\
S_{2, m}=\sum_{j=m}^{s} \frac{(-1)^{j-1}}{(2 j-1) !}\left(\frac{\pi}{2}\right)^{2 j-1} K_{2 s-2 j+1},
\end{gathered}
$$

respectively.

Theorem 24. For $m \geq 2$ and any $s>m$, the following estimates for the truncation errors (82) hold:

$$
\left|S_{1, m}\right|=O\left(\frac{\pi^{2 m}}{(2 m) !}\right), \quad\left|S_{2, m}\right|=O\left(\left(\frac{\pi}{2}\right)^{2 m-1} \frac{1}{(2 m-1) !}\right)
$$

where Landau big O notation is used. For the Landau notation see [27].
Proof. By means of the inequalities (25), it is easy to obtain

$$
\begin{aligned}
\left|S_{1, m}\right| & =\left|\sum_{j=m}^{s} \frac{(-1)^{j-1} \pi^{j p}}{(2 j) !} K_{2 s-2 j+1}\right| \\
& \leq \frac{\pi}{2} \sum_{j=m}^{s} \frac{\pi^{2 j}}{(2 j) !}=\frac{\pi}{2} \frac{\pi^{2 m}}{(2 m) !} \\
& \times\left(1+\frac{\pi^{2}}{(2 m+1)(2 m+2)}+\cdots+\frac{\pi^{2 s-2 m}}{(2 m+1) \cdots(2 s)}\right) \\
& \leq \frac{\pi^{2 m+1}}{2(2 m) !}\left(1+\frac{10}{5 \cdot 6}+\frac{10^{2}}{5^{2} \cdot 6^{2}}+\cdots\right) \\
& \leq \frac{\pi^{2 m+1}}{2(2 m) !}\left(1+\frac{1}{3}+\frac{1}{3^{2}}+\cdots\right) \\
& =\frac{\pi^{2 m+1}}{2(2 m) !} \frac{3}{2} \leq \frac{3 \pi^{2 m}}{(2 m) !} .
\end{aligned}
$$




$$
\begin{aligned}
& \mathrm{m}=12 ; \text { Array }[\mathrm{K}, \mathrm{m}] ; \mathrm{K}_{1}=\frac{\pi}{2} ; \mathrm{d}\left[\mathrm{n}_{-}, \mathrm{x}_{-}\right]:=\frac{\mathrm{x}^{\mathrm{n}}}{\mathrm{n} !} \\
& \text { For }[\mathrm{s}=1, \mathrm{~s} \leq \mathrm{m} / 2, \mathrm{~s}++ \text {, } \\
& \mathrm{K}_{2 \mathrm{~s}+1}=\frac{1}{2}\left((-1)^{\mathrm{s}} \mathrm{d}[2 \mathrm{~s}+1, \pi]+\sum_{\mathrm{p}=1}^{\mathrm{s}} \mathrm{K}_{2 \mathrm{~s}+1-2 \mathrm{p}} \frac{(-1)^{\mathrm{p}-1} \pi^{2 \mathrm{p}}}{(2 \mathrm{p}) !}\right) ; \\
& \mathrm{K}_{2 \mathrm{~s}}=(-1)^{\mathrm{s}} \mathrm{d}\left[2 \mathrm{~s}, \frac{\pi}{2}\right]+\sum_{\mathrm{p}=1}^{\mathrm{s}} \mathrm{K}_{2 \mathrm{~s}+1-2 \mathrm{p}} \frac{(-1)^{\mathrm{p}-1}\left(\frac{\pi}{2}\right)^{2 \mathrm{p}-1}}{(2 \mathrm{p}-1) !} ; \\
& \text { For }\left[s=1, s \leq m, s++, \operatorname{Print}\left[K_{s}, " \quad ", N\left[K_{s}, 30\right]\right]\right] \text {; }
\end{aligned}
$$

Algorithm 1: Mathematica code for exact symbolic and approximate computation with optional 30 digits accuracy of the Favard constants by recursive representations (7a) and (7b).

Consequently, $\left|S_{1, m}\right|=O\left(\pi^{2 m} /(2 m) !\right)$. In the same way for the second truncation sum, we have

$$
\begin{aligned}
& \left|S_{2, m}\right| \leq 2\left(\frac{\pi}{2}\right)^{2 m-1} \frac{1}{(2 m-1) !} \\
& \text { so }\left|S_{2, m}\right|=O\left(\left(\frac{\pi}{2}\right)^{2 m-1} \frac{1}{(2 m-1) !}\right) .
\end{aligned}
$$

By the details of the proof it follows that the obtained representations did not depend on $s$, for any $m<s$. The a priori estimates (83) can be used for calculation of $K_{r}$ with a given numerical precision $\varepsilon$ in order to decrease the number of addends in the sums for larger $s$ at the condition $m<s$.

Remark 25. The error estimates, similar to (83), can be established for other recurrence representations in this paper too.

In Algorithm 1, we provide the simplest Mathematica code for exact symbolic and numerical calculation with optional 30 digits accuracy of the Favard constants $K_{r}$ for $r=1,2,3, \ldots, m$, based on recursive representations (7a) and (7b) for a given arbitrary integer $m>0$. The obtained results are given in Table 1 . We have to note that this code is not the most economic. It can be seen that the thrifty code will take about $2 m^{2}+8 m$ or $O\left(m^{2}\right)$ arithmetic operations in (7a) and (7b).

The basic advantage of using formulas (7a) and (7b) is that they contain only finite number of terms (i.e., finite number of arithmetic operations) in comparison with the initial formula $K_{r}=(4 / \pi) \sum_{\gamma=0}^{\infty}\left((-1)^{\nu(r+1)} /(2 \nu+1)^{r+1}\right)(r=0,1,2, \ldots)$, which needs the calculation of the slowly convergent infinite sum. It must be also mentioned that in Mathematica, Maple, and other powerful mathematical software packages, the Favard constants are represented by sums of Zeta and related functions, which are calculated by the use of Euler-Maclaurin summation and functional equations. Near the critical strip they also use the Riemann-Siegel formula (see, e.g., [28, A.9.4]).

Finally, we will note that the direct numerical integration in (4) is very difficult. This way a big opportunity is given by Theorems 17, 18, and 19 (formulas (65), (67) and (70)) for effective numerical calculation of the classes of multiple singular integrals $\widetilde{A}_{r}(x), \widetilde{B}_{r}(x)$ and $\widetilde{C}_{r}(x)$ for any $x$. Their computation is reduced to find the finite number of the Favard constants $\widetilde{K}_{v}$ for all $v \leq r$ and the calculation of one additional numerical sum. Numerical values for some of the singular integrals are presented in Table 2.

\section{Concluding Remarks}

As it became clear, there are many different ways and welldeveloped computer programs at present, for calculation of the significant constants in mathematics (in particular Favard constants) and for summation of important numerical series. Most of them are based on using of generalized functions as one can see in [2-5]. Nonetheless, we hope that our previously stated approach through integration of Fourier series appears to be more convenient and has its theoretical and practical meanings in the scope of applications, in particular for computing of the pointed special types of multiple singular integrals. The basic result with respect to multiple integrals reduces their calculation to finite number of numerical sums.

\section{Acknowledgments}

This paper is supported by the Bulgarian Ministry of Education, Youth and Science, Grant BG051PO001/3.3-05-0001 "Science and business," financed under operational program "Human Resources Development" by the European Social Fund.

\section{References}

[1] N. Korneǐchuk, Exact Constants in Approximation Theory, vol. 38, chapter 3, 4, Cambridge University Press, New York, NY, USA, 1991.

[2] A. P. Prudnikov, A. Yu. Brychkov, and O. I. Marichev, Integrals and Series: Elementary Functions, chapter 5, CRC Press, Boca Raton, Fla, USA, 1998.

[3] J. Favard, "Sur les meilleurs precedes d'approximation de certaines classes de fonctions par des polynomes trigonometriques," Bulletin des Sciences Mathématiques, vol. 61, pp. 209-224, 1937. 
[4] S. R. Finch, Mathematical Constants, Cambridge University Press, New York, NY, USA, 2003.

[5] J. Bustamante, Algebraic Approximation: A Guide to Past and Current Solutions, Springer Basel AG, Basel, Switzerland, 2012.

[6] S. Foucart, Y. Kryakin, and A. Shadrin, "On the exact constant in the Jackson-Stechkin inequality for the uniform metric," Constructive Approximation, vol. 29, no. 2, pp. 157-179, 2009.

[7] Yu. N. Subbotin and S. A. Telyakovskiü, "On the equality of Kolmogorov and relative widths of classes of differentiable functions," Matematicheskie Zametki, vol. 86, no. 3, pp. 432-439, 2009.

[8] I. H. Feschiev and S. G. Gocheva-Ilieva, "On the extension of a theorem of Stein and Weiss and its application," Complex Variables, vol. 49, no. 10, pp. 711-730, 2004.

[9] R. A. DeVore and G. G. Lorentz, Constructive Approximation, vol. 303, Springer, Berlin, Germany, 1993.

[10] V. P. Motornyı̆, "On sharp estimates for the pointwise approximation of the classes $W^{r} H^{\omega}$ by algebraic polynomials," Ukrainian Mathematical Journal, vol. 53, no. 6, pp. 916-937, 2001, Translation from Ukrains'kyi Matematychnyi Zhurnal, vol. 53, no. 6, pp. 783-799, 2001.

[11] W. Xiao, "Relative infinite-dimensional width of Sobolev classes $W_{p}^{r}(R)$, , Journal of Mathematical Analysis and Applications, vol. 369, no. 2, pp. 575-582, 2010.

[12] P. C. Nitiema, "On the best one-sided approximation of functions in the mean," Far East Journal of Applied Mathematics, vol. 49, no. 2, pp. 139-150, 2010.

[13] O. L. Vinogradov, "Sharp inequalities for approximations of classes of periodic convolutions by subspaces of shifts of odd dimension," Mathematical Notes, vol. 85, no. 4, pp. 544-557, 2009, Translation from Matematicheskie Zametki, vol. 85, no. 4, pp. 569-584, 2009.

[14] A. V. Mironenko, "On the Jackson-Stechkin inequality for algebraic polynomials," Proceedings of Institute of Mathematics and Mechanics, vol. 273, supplement 1, pp. S116-S123, 2011.

[15] V. F. Babenko and V. A. Zontov, "Bernstein-type inequalities for splines defined on the real axis," Ukrainian Mathematical Journal, vol. 63, pp. 699-708, 2011.

[16] G. Vainikko, "Error estimates for the cardinal spline interpolation," Journal of Analysis and its Applications, vol. 28, no. 2, pp. 205-222, 2009.

[17] O. L. Vinogradov, "Analog of the Akhiezer-Krein-Favard sums for periodic splines of minimal defect," Journal of Mathematical Sciences, vol. 114, no. 5, pp. 1608-1627, 2003.

[18] L. A. Apaĭcheva, "Optimal quadrature and cubature formulas for singular integrals with Hilbert kernels," Izvestiya Vysshikh Uchebnykh Zavedeniu, no. 4, pp. 14-25, 2004.

[19] F. D. Gakhov and I. Kh. Feschiev, "Approximate calculation of singular integrals," Izvestiya Akademii Nauk BSSR. Seriya Fiziko-Matematicheskikh Nauk, vol. 4, pp. 5-12, 1977.

[20] F. D. Gakhov and I. Kh. Feschiev, "Interpolation of singular integrals and approximate solution of the Riemann boundary value problem," Vestsī Akadèmīi Navuk BSSR. Seryya FìzikaMatèmatychnykh Navuk, no. 5, pp. 3-13, 1982.

[21] B. G. Gabdulkhaev, "Finite-dimensional approximations of singular integrals and direct methods of solution of singular integral and integro-differential equations," Journal of Soviet Mathematics, vol. 18, pp. 593-627, 1982.

[22] V. P. Motornyı̆, "Approximation of some classes of singular integrals by algebraic polynomials," Ukrainian Mathematical Journal, vol. 53, no. 3, pp. 377-394, 2001.
[23] E. Vainikko and G. Vainikko, "Product quasi-interpolation in logarithmically singular integral equations," Mathematical Modelling and Analysis, vol. 17, no. 5, pp. 696-714, 2012.

[24] H. Brass and K. Petras, Quadrature Theory: The Theory of Numerical Integration on a Compact Interval, vol. 178, American Mathematical Society, Providence, RI, USA, 2011.

[25] E. W. Weisstein, "Favard constants," http://mathworld.wolfram .com/FavardConstants.html.

[26] A. G. Zygmund, Trigonometric Series, vol. 1, Cambridge University Press, 2nd edition, 1959.

[27] D. E. Knuth, The Art of Computer Programming. Vol. 1: Fundamental Algorithms, Section 1.2.11: Asymptotic Representations, Addison-Wesley, 3rd edition, 1997.

[28] S. Wolfram, The Mathematica Book, Wolfram Media, Champaign, Ill, USA, 5th edition, 2003. 


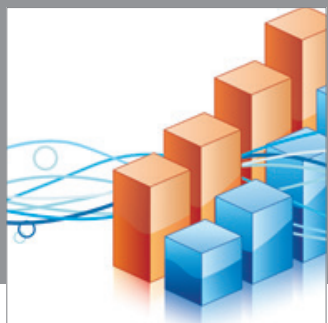

Advances in

Operations Research

mansans

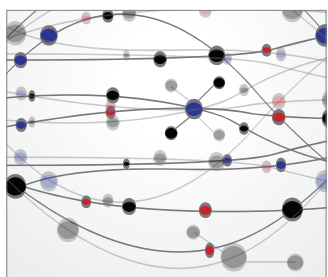

The Scientific World Journal
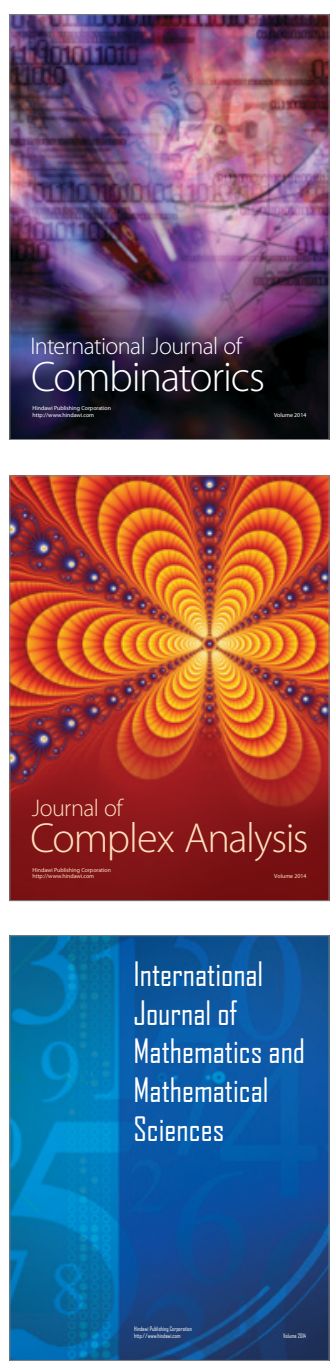
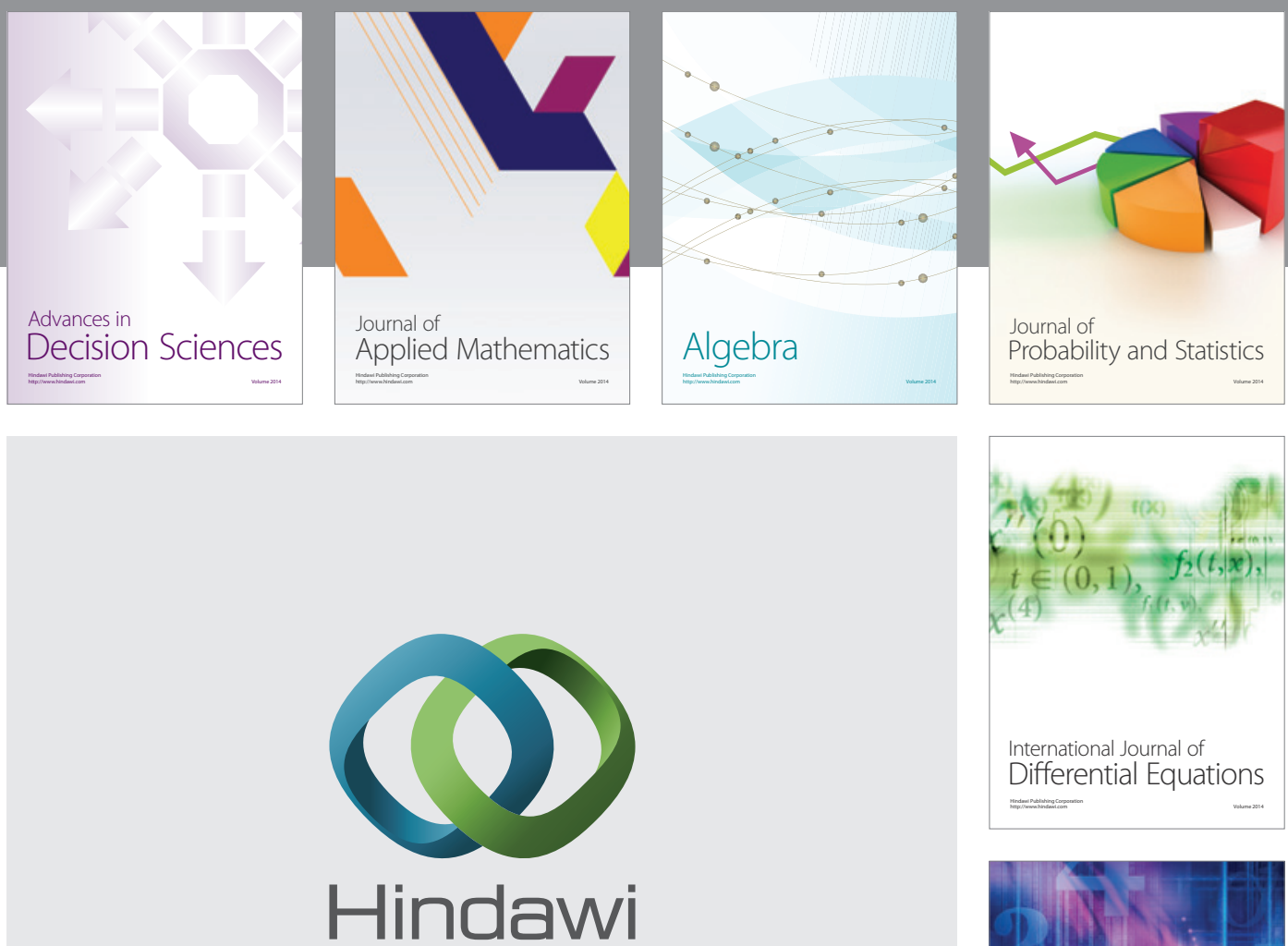

Submit your manuscripts at http://www.hindawi.com
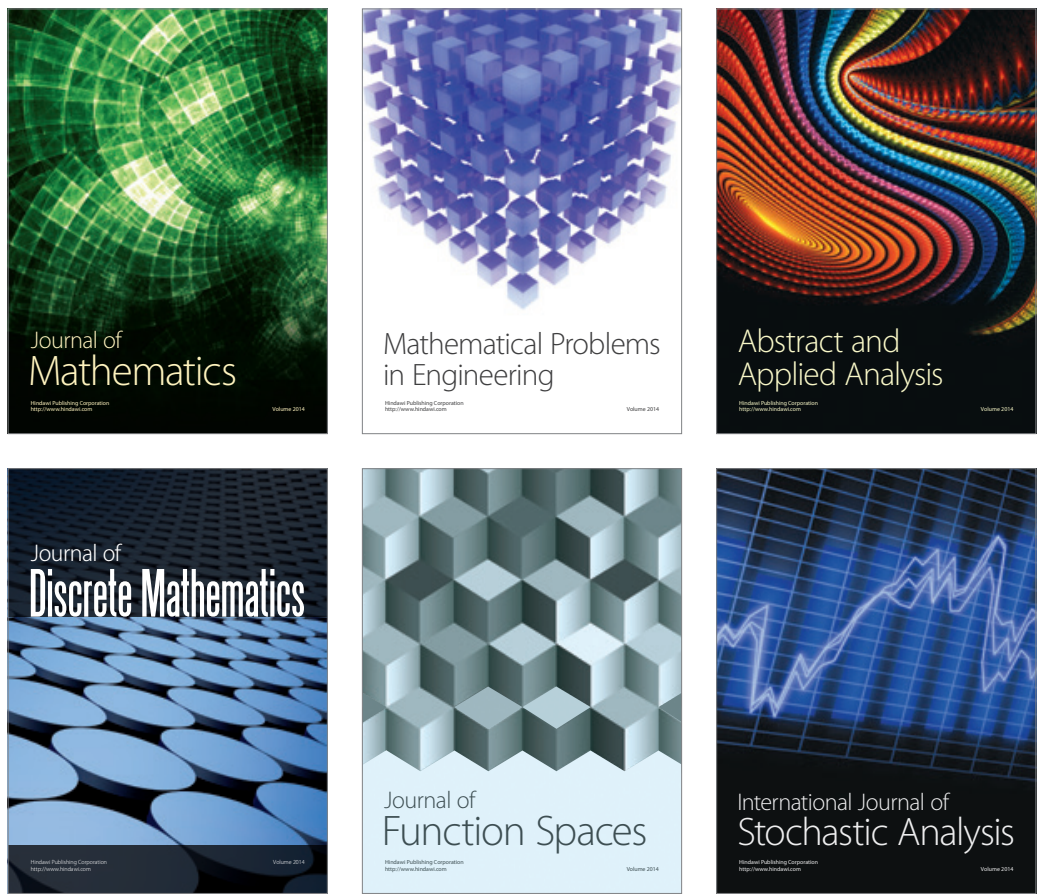

Journal of

Function Spaces

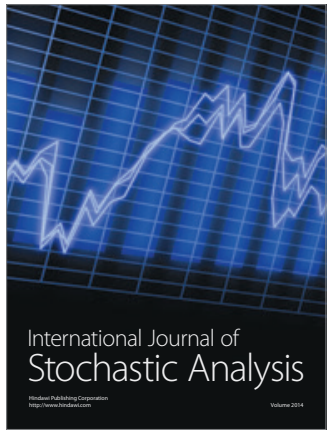

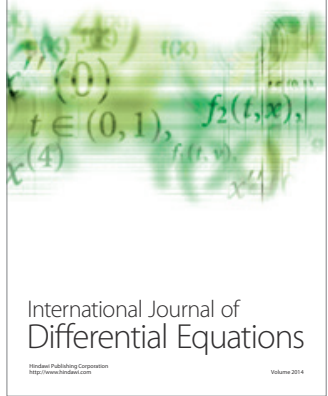
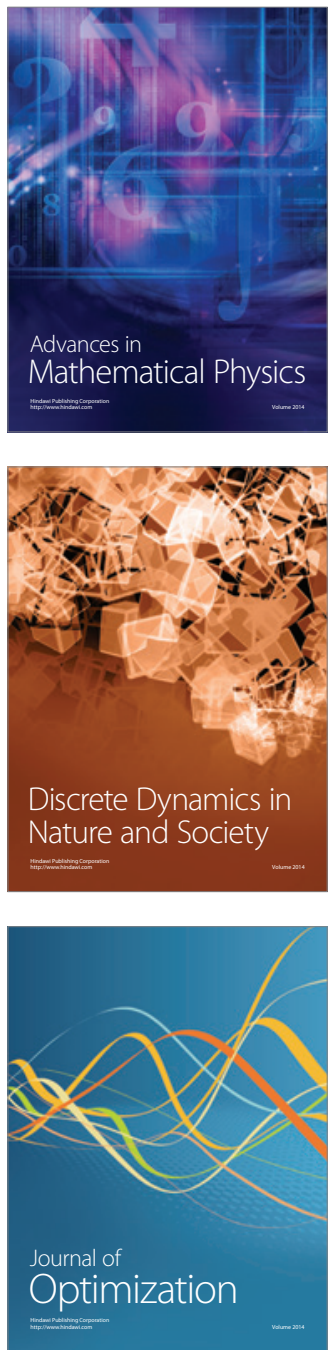